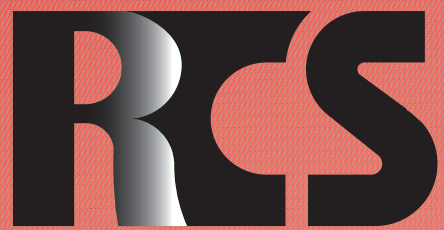

Depósito legal ppi $201502 Z U 4662$

Esta publicación científica en formato digital es continuidad de la revista impresa Depósito Legal: pp $197402 Z U 789$

- ISSN: 1315-9518 • ISSN-E: 2477-9431

Revista de Ciencias Sociales

Universidad del Zulia. Revista de la Facultad de Ciencias Económicas y Sociales Vol. XXVII. No. 2

Abril-Junio 2021

Esta publicación científica en formato digital es continuidad de la revista impresa Depósito Legal: pp $197402 Z$ Z789 ISSN: 1315-9518 


\title{
Turismo rural en Crucita-Ecuador: Una mirada desde la fortaleza del género
}

\author{
Bumbila García, Bibian Bibeca*
}

\section{Resumen}

En la actualidad, un grupo de investigaciones en todo el mundo se enfocan en el esbozo de estrategias para el desarrollo del turismo como eje potenciador de las economías. En ese sentido, la presente investigación se direcciona en realizar un análisis sobre un proyecto de turismo en el espacio rural con enfoque de género y que potencie el desarrollo comunitario, en la parroquia Crucita, perteneciente al Cantón Portoviejo, provincia ecuatoriana de Manabí-Ecuador. El estudio a nivel metodológico parte de un enfoque postpositivista, con el paradigma interpretativo. El análisis de la disquisición realizada revela que aun cuando en Crucita se aprecian avances en cuanto a la resiliencia y autonomía femenina, sin embargo, persisten elementos propios de una cultura patriarcal y machista que dificultan su visibilización, es decir, el no querer concebir totalmente la presencia de la mujer como figura relevante en las labores relacionadas con la gastronomía y en otros sectores de la economía, que en ciertos momentos la limitan en su pleno desarrollo en la sociedad. Se concluye, que de modo general, se reconoce la importancia de implementar en la localidad iniciativas donde la mujer promocione sus talentos dentro del área de la gastronomía, propiciando así su participación social.

Palabras clave: Turismo rural; desarrollo comunitario; gastronomía; enfoque de género; Crucita-Ecuador.

Doctorante en Ciencias Sociológicas. Desarrollo Social Comunitario (Cuba). Doctora en Ciencias de la Educación mención Pedagogía. Magister en Gerencia y Liderazgo Educacional. Licenciada en Ciencias de la Educación, especialidad Psicología y Orientación Vocacional. Docente Titular a Tiempo Completo de la Universidad Técnica de Manabí, Ecuador. E-mail: bibian.bumbila@utm.edu.ec iD ORCID: https://orcid.org/0000-0001-8928-3219 


\title{
Rural tourism in Crucita-Ecuador: A look from the strength of the gender
}

\begin{abstract}
Currently, a group of researches around the world are focused on the outlining of strategies for the development of tourism as a driving force behind economies. In this sense, this research is aimed at carrying out an analysis on a tourism project in rural areas with a gender perspective and that promotes community development, in the Crucita parish, belonging to the Portoviejo Canton, Ecuadorian province of ManabíEcuador. The study at the methodological level starts from a postpositivist approach, with the interpretive paradigm. The analysis of the disquisition carried out reveals that even when Crucita shows progress in terms of female resilience and autonomy, however, elements of a patriarchal and macho culture persist that make it difficult to make visible, that is, not wanting to fully conceive of the presence of women as a relevant figure in work related to gastronomy and in other sectors of the economy, which at certain times limit their full development in society. It is concluded that, in a general way, the importance of implementing initiatives in the locality where women promote their talents within the gastronomy area is recognized, thus promoting their social participation.
\end{abstract}

Keywords: Rural tourism; community development; gastronomy; Gender approach; Crucita-Ecuador.

\section{Introducción}

En la actualidad, un grupo de investigaciones en todo el mundo se enfocan en el esbozo de estrategias para el desarrollo del turismo como eje potenciador de las economías. Resulta que, sin llegar a cauces ostentosos, es posible sustentar que la también llamada industria del ocio, o sin humo, desempeña un papel esencial para el desarrollo y la dinamización del tejido socioeconómico de las naciones.

No en vano, el turismo internacional tuvo un crecimiento del $4 \%$ registrado en el primer trimestre de 2019, según lo promulgado en la última edición del Barómetro OMT de Turismo Mundial, elaborado por la Organización Mundial del Turismo (OMT, 2019), denominado ese año, del "Empleo, habilidades y trabajo" tras reconocer la capacidad del sector para generar más y mejores empleos incluyentes; las cifras señalan que el turismo provee una de cada diez plazas de trabajo en todo el mundo; así también representa el 10\% del PIB mundial; lo que demuestra el aporte positivo al desarrollo; sin embargo, se requiere afianzar compromisos entre los países para continuar con el crecimiento de la actividad turística de forma sostenible.

Ecuador, no se encuentra ajeno a estas realidades, al sobresalir como uno de los referentes en la región, al recibir millones de turistas anualmente, seducidos por la extensa riqueza natural y cultural del país, que acumula destinos heterogéneamente diversos, entre sitios históricos, espacios rurales o zonas de costas, sol y playa, en distintas regiones.

Las regiones turísticas, pueden ser inscritas en el concepto más amplio de la economía, de allí, que se le considere al turismo una industria, porque bien utilizados 
los atractivos que un país tiene con su naturaleza, espacios de esparcimiento, sitios históricos, los cuales se muestran con orgullo por ser parte de esa nación, generan un ingreso importante bien sea para la localidad, la región o el país, manifestando Pelegrín, et al. (2020), que están caracterizadas:

Por el predominio de este tipo de actividad, a la cual se dirigen los mayores esfuerzos, tanto de las relaciones que se establecen entre las diversas fuerzas productivas, como los intereses del sistema determinado por las relaciones de producción establecidas. (p.232)

En tal sentido, esta industria turística permite resaltar lo bonito, atractivo de una localidad, a veces patrimonio nacional, pero no solo eso es lo importante, sino que se convierte en un punto específico para el desarrollo local, sostenible y sustentable en algunos casos, porque la gente aprovecha esas maravillas de la naturaleza, esa cultura y costumbre del lugar, para generar ingresos que a su vez se convierten en empleos para los lugareños y reinvertir en el espacio turístico.

Es cierto que el turismo, es una actividad temporal para algunos lugares y depende de la mercadotecnia para hacer de ese lugar un espacio frecuentado, por lo cual, muchas personas han pensado en esto como parte de sus actividades específicas cuando es temporada de vacaciones, fines de semana larga, o eventos culturales que permiten resaltar esa zona.

Aunado a este punto importante del turismo, que se convierte en un gran reto, aparejado al desarrollo turístico del país, está el diseño de proyectos que asuman las cuestiones referentes a la igualdad de género y que consideren, a la par de un desarrollo local sostenible, la necesidad de incorporar a las mujeres, muy especialmente las que habitan en esas regiones, como protagonistas de ese crecimiento y con igualdad de derechos, oportunidades, remuneración y acceso a las actividades propias del sector; por lo cual, Pérez, et al. (2020) manifiestan que es necesario "realizar una gestión sostenible del destino tanto por las administraciones públicas como por los gestores privados" (p.20).
Urge entonces, visualizar en qué medida puede hablarse de un turismo rural basado en relaciones desiguales, a partir del estudio exhaustivo de los fenómenos que suceden en esos espacios y de qué manera las mujeres se ven beneficiadas o no de la obtención de ganancias, tomando en consideración el funcionamiento a lo interno de muchas familias, donde el hombre zanja a su voluntad la repartición de los dividendos recibidos.

No obstante, como paradoja distintiva se observan las políticas implementadas por el Estado para la consecución de la igualdad de género y el respeto a la diversidad, así se dispone, entre otros estatutos, en el artículo 11, numeral 2 de la Constitución de la República del Ecuador 2008 (Asamblea Nacional, 2011), que ahonda en la no discriminación por razones de sexo, recalcando las mismas oportunidades para la mujer que para el hombre, en el campo del respeto laboral, haciéndose énfasis que lo que puede hacer bien uno lo puede hacer también el otro, es decir, que la actividad no está detallada por género, como lo es en este caso, el turismo.

Asimismo, se visibiliza el interés por la incorporación de la perspectiva de género como vía expedita para desmontar lascivos rezagos machistas, transmitidos de generación en generación, que sin dudas dan paso a la conformación de patrones de conducta que atentan contra el desarrollo íntegro de la mujer con su aportación en proyectos de desarrollo $\mathrm{y}$ al auge del turismo como fuerza emergente.

En el presente estudio, amparado en el paradigma interpretativo, se parte entonces de pensar en la acción comunitaria desde el género, por lo cual, se propone realizar un diagnóstico de la situación de la parroquia Crucita, perteneciente al cantón Portoviejo, ubicado en la provincia de Manabí, Ecuador; con el propósito de establecer las directrices fundamentales a tomar en consideración para el encauzamiento de un proyecto de turismo en el espacio rural con enfoque de género, que potencie el desarrollo local. Se toma la posición de Trejo, Llaven y Pérez (2015), cuando asumen una definición de la web sobre enfoque de género que: 
Considera las diferentes oportunidades que tienen los hombres y las mujeres, las interrelaciones existentes entre ellos y los distintos papeles que socialmente se les asignan. Todas estas cuestiones influyen en el logro de las metas, las políticas y los planes de los organismos nacionales e internacionales $\mathrm{y}$ por lo tanto, repercuten en el proceso de desarrollo de la sociedad. (p.6)

Expresándose lo anterior, porque en el campo del turismo, como en cualquier otro, la mujer puede realizar sus actividades como proyecto propio, según sus competencias, buscando que su trabajo se convierta en algo formal y aceptado con el mismo rigor que si lo hiciera un hombre, porque tienen las mismas oportunidades y capacidades.

Para tan ambicioso objetivo, resulta esencial entender que, si bien existe un proyecto de desarrollo cantonal, cimentado en la filosofía del Buen Vivir, los mecanismos para alcanzar sus objetivos, obvian lo referente a las problemáticas de género, lo cual, sin proponérselo, multiplican y afianzan la exclusión social, producto de la adopción de criterios heredados de una cultura patriarcal y machista, asentada durante siglos en las zonas rurales de Ecuador y de la región latinoamericana de modo general, donde la actividad laboral de la mujer se considera apenas un complemento de la del hombre.

Con base en la explicación, se parte del escenario investigado, Crucita, un balneario turístico, ubicado geográficamente en el Valle del Río Portoviejo, en la costa de la provincia de Manabí. La parroquia poseía en el 2010 más de 14.050 habitantes, según datos del Instituto Nacional de Estadística y Censo (INEC, 2010), ratificándose en el Plan de Desarrollo y Ordenamiento Territorial (PDOT) de la Parroquia Crucita (Secretaría Nacional de Planificación y Desarrollo [SENPLADES], 2015), que es la más poblada del cantón. Llama la atención, que las propias cifras indican que la cantidad de hombres era superior a la de mujeres (7.184 por 6.866), y cerca del $73 \%$ de las féminas se encontraban dentro de la población inactiva desde el punto de vista laboral (no tienen trabajo formal y tampoco están buscándolo).

Una vez expuestos los elementos anteriores, emerge una idea central a investigar: La necesidad de promover proyectos turísticos centrados en la equidad de género, donde se aprovechen las potencialidades del territorio, cuyos resultados se reviertan en beneficios para todos los actores de la comunidad. De ahí surge la pregunta: ¿Qué aspectos posee el desarrollo local a través del turismo en el espacio rural de la parroquia Crucita, Portoviejo, desde una perspectiva de género?

Asimismo, se persiguen los siguientes objetivos: De manera general, analizar la situación de la parroquia Crucita, del cantón Portoviejo, provincia ecuatoriana de Manabí, para fundamentar un proyecto de turismo en el espacio rural con enfoque de género con el cual se potencie el desarrollo comunitario. Este propósito se apoya en dos objetivos específicos: 1) Determinar los fundamentos sociológicos de las articulaciones entre turismo en el espacio rural, género y desarrollo comunitario en la parroquia Crucita; 2) constatar la importancia del esbozo de proyectos de desarrollo cimentados en el turismo y con enfoque de género en la parroquia Crucita.

Con base en el planteamiento expuesto, en el presente artículo se propone, un análisis de estas temáticas, tomando en consideración el posible impacto de un proyecto verdaderamente inclusivo y las posibilidades de la imbricación en él de la gastronomía como actividad fundamental, considerando a la mujer el eje central de esta actividad turística.

\section{Turismo, desarrollo local y género: La visión teórica}

El término desarrollo local, puede apreciarse desde diversas perspectivas y a partir de multiplicidad de criterios. Precisamente, esas perspectivas varían entre visiones meramente teóricas hasta otras que lo asumen a través de iniciativas propias para su fomento. Segrelles (2015), opina que se caracteriza por estar constituido por sistemas locales de actores, pero no se debe a la localización 
del desarrollo en un lugar determinado, por tanto, "las acciones de desarrollo pueden ser caracterizadas como desarrollo local cuando generan o refuerzan dinámicas sistémicas de escala local" (p.1), de esta configuración se asume el rol activo del territorio, cuyo origen se debe a la pertenencia de los actores.

El desarrollo local es, ciertamente, un medio de transformación de la economía y la sociedad de esos espacios, que repercute directamente en una mejor calidad de vida para sus habitantes. Puede entenderse ese desarrollo, a partir de su importancia como impulso de la economía, agente de dinamismo en la sociedad, y creador de espacios laborales y riqueza destinadas a satisfacer las necesidades de lugareños. Resulta imprescindible la sinergia, entre los actores para el florecimiento de los proyectos que se coloquen en marcha, lo cual, entraña modificaciones conductuales y de proceder de las entidades, los grupos y las personas de modo general.

Mencionan Verdesoto y Ardaya (2011), que "una forma de entender el desarrollo local y territorial de un país consiste en apelar a los desequilibrios económicos del territorio como clave analítica y de la política pública" (p.142); por ello, no se puede negar las diferencias de renta entre las regiones, provincias y cantones, pero a pesar de eso, no puede dejarse anclar el desarrollo local y territorial en la búsqueda de igualdad con respecto a los subsidios, las asignaciones discrecionales, las compensaciones, condenando a los territorios a una reproducción con relación asistida por la nación y no una incorporación ciudadana de los territorios a los factores de la nación, de la sociedad y del Estado, así como al mercado nacional.

No cabe duda que para lograr el desarrollo local, territorial, es necesaria la participación social y ciudadana, responsable y comprometida de los lugareños, quienes desde su espacio pueden construir así como acumular el capital social y simbólico, identidad territorial, robusteciendo con su trabajo la institucionalidad local, donde se produce con sentido solidario para el bienestar local y del país en general aprovechándose las bondades de Crucita, con sus playas atractivas. Al respecto, Formichella (2004) considera que el desarrollo local, cimentado en el turismo, es dinámico e integrador y debe presentársele especial atención a líneas de desarrollo enfocadas en sus elementos económicos, ambientales, sociales, culturales, políticos y tecnológicos. Precisamente entre el potencial de desarrollo, se hace factible el fomento del turismo como factor de peso, para lo cual se utilizan de manera oportuna todos los recursos presentes en el territorio.

Además de la potenciación de la participación social, con el desarrollo local, debe promoverse la formación de organizaciones intermedias para la provisión de servicios públicos y la conformación de empresas de pequeña escala, basadas en demandas genuinas y modos de producción sostenibles y sustentables, tomando en cuenta la necesidad de proponer soluciones al problema desde lo local, se puedan plantear soluciones a problemas globales, como proceso endógeno de cambio estructural para propiciar el empoderamiento de la sociedad local, a través de la descentralización política y la devolución de poderes a las autoridades locales.

Pero para lograrlo es importante saberlo hacer, y es aquí donde la formación de la ciudadanía es necesaria de manera que puedan hacerse garantes de que no se produzca una total separación entre objetivos de crecimiento económico y límites del desarrollo humano. Para ello, las universidades están en el compromiso de generar programas de especialización, maestría, doctorado que doten a sus participantes de las competencias sobre desarrollo en los territorios y las dinámicas que lo permiten, con la finalidad de que planteen estrategias y brinden soluciones a las necesidades, problemas y aspiraciones de cada localidad, de forma que se intervenga estratégicamente.

Por otra parte, el desarrollo local difiere, según las particularidades de cada país y las sui géneris maneras como funcionen las sociedades, aunque de modo general, toma como eje fundamental al ser humano y los 
propósitos colectivos. De modo que, requiere la participación de todos por igual para su aseguramiento.

\subsection{Nociones sobre el desarrollo humano: Perspectivas desde lo local}

Reflexionar sobre los paradigmas del desarrollo desde el punto de vista sociológico, y de los referentes teóricos que signan su conceptualización, resulta esencial para la comprensión integral de ese fenómeno. Es preciso partir de los preceptos de Pacheco, Carrera y Almeida (2011), quienes destacan como aspectos inherentes a la concepción de proyectos turísticos enfocados hacia el desarrollo "evaluar indicadores socioculturales, económicos-financieros, ambientales y turísticos, aspectos inherentes a la actividad turística y de importancia para identificar fortalezas y debilidades de las comunidades que puedan ser mejoradas" (p.44). Al respecto, Monge y Macías (2016) sostienen que existe el Desarrollo Local Integrado el cual:

Haceespecial hincapiéen lacombinación de todas las potencialidades existentes en la zona. En este modelo, se intenta minimizar la utilización de tecnologías externas, poniendo especial énfasis en la movilización y el desarrollo de los recursos humanos y en la consecución de un acceso más equitativo a los medios de producción y a una más justa distribución de la renta. (p.229)

En ese sentido, hacen énfasis los autores precitados en la importancia de considerar al recurso humano de ese espacio, para poder generar desde lo endógeno, el bienestar de la comunidad, mediante el establecimiento de actividades económicas y socioculturales, utilizando fundamentalmente sus propios recursos humanos y materiales, donde la iniciativa privada adquiere un papel preponderante, por lo que se requieren nuevas fórmulas de colaboración empresarial y de participación social, que serán los mismos protagonistas del proyecto, quienes buscaran esa colaboración y alianzas.

No obstante, resulta preciso señalar que desarrollo humano debe entenderse más allá de las superfluas ideas que lo asumen solo a través de estadísticas como ingreso per cápita, pues comprende una serie de procesos que además de crecimiento y productividad, incluyen también la sustentabilidad, participación, equidad de género, la etnicidad, entre otras categorías. Al respecto, consideran Tezanos, et al. (2013) que:

El desarrollo humano es un proceso de progreso forjado por las personas, y que beneficia a las personas, las políticas de desarrollo humano (nacionales e internacionales) deben diseñarse de manera participa (empoderando a las personas), y sus beneficios deben distribuirse de manera equitativa ( $\sin$ distinción de nacionalidad, sexo, etnia, edad, religión, o condición física o mental), abordando con justicia los potenciales conflictos que surgen en una comunidad (la humana) en constante crecimiento, pero que habita en una planeta finito y compartido. (p.12)

Como se entiende, el desarrollo humano abarca muchos aspectos tomando en cuenta la posibilidad de cualquier persona según sus conocimientos y habilidades de empoderarse para lograr algo determinado, que en este caso, sería un proyecto turístico en Crucita. Lo cierto es que, el desarrollo ha sido visualizado históricamente desde disímiles enfoques, y perspectivas. Resulta preciso destacar, que analíticamente deviene un concepto complejo y con matiz multidimensional. El Programa de las Naciones Unidas para el Desarrollo (PNUD, 2006), insiste en que:

Sus dimensiones constitutivas son: la económica: referida a la creación, acumulación y distribución de riqueza, la economía sustentable, y el bienestar común. La social y cultural: referida a la calidad de vida, a la equidad, a la identidad cultural y a la integración social. La territorial: referida al territorio como agente del desarrollo; construcción de territorios prósperos, democráticos $\mathrm{e}$ integrados. La ambiental: referida a los recursos naturales y a la sustentabilidad de los modelos de desarrollo adoptados en el mediano y largo plazo. La política: 
referida a la gobernabilidad del territorio $\mathrm{y}$ a la definición de un proyecto colectivo específico, autónomo y sustentado en los propios actores locales. (p.13)

Un análisis exhaustivo de las ideas antes expuestas, lleva a concordar con el PNUD (2006), cuando lo visualiza como la manera de ampliar las oportunidades del ser humano, las cuales son infinitas con modificación en el tiempo, pero que propicia tres aspectos esenciales como son el disfrutar de una vida prolongada y saludable; adquirir conocimientos y tener acceso a los recursos necesarios para lograr un nivel de vida digno, sabiéndose que se trabaja por algo y para algo, lo cual le da fortaleza.

Por otra parte, resulta imprescindible comprender las barreras propias entre los fundamentos relativos al desarrollo comunitario y local, pues coexistir en el mismo espacio geográfico, no se traduce inexorablemente en relaciones sociales cuyo propósito esencial sea alcanzar determinado desarrollo. Sobre esto, Alonso, Riera y Rivero (2013) explican que:

El término comunidad, aunque pueda designar correctamente el vínculo realmente existente entre personas del lugar (en términos estructurales), no significa que tales vínculos tengan el desarrollo pleno que hoy resulta posible y necesario dentro de la lógica de proyectos sociales emancipadores. (p.1)

Emana entonces como soporte primordial de la presente investigación, la comprensión de la importancia de las relaciones sociales e interacciones que suceden en el seno de determinado territorio para un verdadero desarrollo local, como es Crucita, siendo imprescindible los vínculos entre los actores de la comunidad y la participación de estos en todos los procesos.

\subsection{El turismo como potenciador del desarrollo local}

Cuando se habla de turismo, se está refiriendo a la actividad de personas que visitan y disfrutan de los lugares más importantes de una región, mencionando Zambrano, Alejo y Zambrano (2019) que "el turismo es toda actividad asociada a las personas y sus motivaciones: El ocio, el descanso y el deseo de descubrir nuevos lugares" (p.201); por ello, la Organización Mundial del Turismo (2019) plantea que es posible comprenderlo como un fenómeno social, cultural y económico, vinculado con el movimiento de personas a sitios fuera de su lugar de residencia habitual por razones personales, de ocio, comerciales o de otra índole.

Al turismo se representa como esa acción de estar en un lugar que no es su casa, y solo la visita de manera temporal. En este sentido, los atractivos que pueden cautivar al visitante, pueden ser muy diversos como las zonas, bienes, tradiciones y acontecimientos, que por sus particularidades o ubicación constituyen objetos de interés. A su vez, como fenómeno comprende un sinnúmero de actividades adicionales, y su carácter transversal está dado por la manera en que su propio desarrollo influye también en otras esferas y negocios.

Al respecto del turismo rural, este irrumpe como elemento esencial para la presente investigación, de allí que como lo mencionan Millán, Morales y Pérez (2014), es oportuno entenderlo como instrumento para alcanzar el desarrollo en zonas signadas por un marcado desequilibrio económico. Lo expuesto implica entender que si es un lugar visitado en épocas del año, y la economía está basada en la producción que se desarrolla con la visita de los turistas, siempre es necesario asumir proyectos que le permitan mantenerse dinámica y permanentemente en producción, de manera que se beneficie la zona y repercuta en el progreso de la región y del país, pues si bien el sector está considerado como fuente de desarrollo y crecimiento económico en esos espacios, de igual manera puede multiplicar la desigualdad. Explica García (2016):

Muchos de los programas de turismo comunitario tienen una repercusión mínima o nula sobre las prácticas locales de uso de recursos, mejoran solo modestamente los ingresos familiares y dependen excesivamente de ayudas externas no solo a corto, sino también a largo plazo, y a veces 
indefinidamente. (p.611)

Respecto a las características que particularizan al turismo rural, según Millán, et al. (2014), se encuentran que puede devenir en elemento de estabilización sociodemográfica, al impedir el éxodo de población de zonas rurales a urbanas, produciendo a su vez, una disminución del desempleo en aquellas ciudades con alta migración rural. Asimismo, no depende de la estacionalidad que marca al sector; provoca pluriactividad en la población de las áreas rurales; y en muchas ocasiones, trae consigo el aliciente y parte de la inversión necesaria para la creación de infraestructuras requeridas para el desarrollo de otras actividades económicas. Para Flores y Barroso (2012):

En una sociedad desarrollada donde se producen cambios en la economía las zonas rurales buscan alternativas que reduzcan la excesiva dependencia de las actividades agrícolas y ganaderas y que, al mismo tiempo, permitan adaptar sus estructuras productivas a las exigencias de esta nueva sociedad del ocio y del tiempo libre. (p.62)

En ese sentido, se considera que las opciones productivas de las cuales disponen las administraciones locales permitan lograr la reconversión económica, posiblemente, donde el turismo es una de las que más éxito pueden tener, tomándose en cuenta los elementos positivos, aunque en la actualidad, se estén visibilizando problemas para el desarrollo del turismo rural.

Por lo tanto, la idea de turismo en el espacio rural de por sí no aporta un campo conceptual verdaderamente nítido. Es por ello, que resulta posible distinguir entre turismo rural y turismo en el espacio rural, porque según De Oliveira, et al. (2014) el turismo rural, es aquel que tiene a lo rural como medio para generarse, en cambio aquel que se desarrolla en el espacio rural, se caracteriza por no encontrarse relacionado directamente con la visita a senderos o la actividad agropecuaria, aunque tenga lugar en un espacio rural, sino que se llevan a cabo otras actividades.

Sin embargo, lo importante es que si se toman esos espacios rurales, con su cultura, costumbres y actividades de agricultura, ganadería, pesca, sus playas, montañas, los sitios históricos, patrimonios nacionales o mundiales, permitiendo las posibles intervenciones de la administración pública, sea comunitaria, nacional y regional, se puede favorecer el desarrollo turístico de estas zonas, generándose la contribución de la oferta turística nacional y regional, basada en el turismo de sol y playa, como es el caso de Crucita.

De modo general, cada vez con mayor fuerza se relaciona el desarrollo local con el turismo, por las grandes oportunidades que presenta, como trabajo dinámico de la misma gente del lugar, cuyas actividades deben aplicarse como motor de desarrollo, por lo cual, su reto como actividad económica es lograr la motivación primordial en la población local para la participación eficaz en ese proceso.

\subsection{Enfoque de género: Perspectivas de participación de la mujer en el turismo rural y los proyectos de desarrollo}

Dentro de las ideas del estudio, es pertinente exponer que el Instituto Internacional de Investigación y Capacitación para la Promoción de la Mujer (INSTRAW, 2006), asume el género como los distintos roles, actitudes, comportamientos, valores, poder relativo e influencia, que la sociedad asigna a ambos sexos de forma diferenciada. Esta idea lleva por el camino de entender el género como construcción simbólica, que va más allá de lo biológico, deviene estructuras asentadas durante años en las mentes, como interpretación cultural de lo que significa ser hombre o mujer en la sociedad.

Las desigualdades de género existentes en América Latina, han sido definidas por numerosos estudios como aspecto a considerar para el esbozo de iniciativas que fomenten un crecimiento local, tomando en cuenta entre tantas, la característica de machismo que condiciona la actuación del hombre sobre la mujer y que ésta en lugar de evitarlo, lo resalta 
más, al darle poder al otro, sin entender que ella si puede llevar a cabo proyectos, porque tiene las capacidades para hacerlo.

Entonces, ¿Es posible acoplar un enfoque de género a los proyectos de desarrollo local cimentados en el turismo?; al respecto, expresa Serrano (2012) que el enfoque de género, lo sustenta enmarcado en los postulados de expertos, "es que propone y permite el análisis profundo de las relaciones sociales entre hombres y mujeres, con el fin de esclarecer las diferencias e inequidades en salud que están vinculadas al género" (p.4). Por lo tanto, visualizar el desarrollo local desde el género, posibilita el análisis profundo, no solo de la problemática de la mujer, sino de las disímiles desigualdades que subsisten pero que analizando las fortalezas posibles, permite grandes oportunidades, explicando Ferguson (2010) que el género:

En las políticas de turismo se beneficiara de un debate más abierto y honesto sobre el tipo de "empoderamiento" deseable y factible. A juzgar por los indicios mostrados, el empoderamiento económico a través del trabajo en el turismo no tiende a incidir de manera significativa en las relaciones de poder que subyace a una relativa mejora de las condiciones económicas. (p.133)

De allí, que mantener un debate abierto sobre la participación de un proyecto turístico dirigido por mujeres, permitirá que las cuestiones de género y las políticas de turismo acerca de esto, propiciarían la oportunidad de reconsiderar los valores y los procesos, por cuanto las ideas que aporta el feminismo tienen un importante papel en este sentido, y ofrecen el potencial para una reformulación de los debates sobre las políticas a colocar en marcha y agilizar un cambio social transformador.

Manifiestan Pérez y Fuller (2015), que incorporar estas nociones a propuestas que tomen como base el turismo, resulta, más que posible, necesario, pues precisamente los estigmas de capacidades y actitudes respecto al género, son los que sitúan a las mujeres en funciones ligadas a la reproducción social, mientras los hombres son vistos como gerentes o cabezas de empresa.

En razón de lo expuesto clarifica entonces, que contemplar un verdadero enfoque de género en un proyecto, implica necesariamente romper con los cánones machistas heredados de generación en generación, donde se reproducen las acostumbradas relaciones de poder entre uno y otro sexo. Señala Rodríguez (2011), que "en muchos aspectos de la vida se mantienen abierta y sutilmente, el modelo social, cultural y familiar del poder masculino y la subordinación de la dependencia femenina" (p.4).

En las iniciativas de turismo en el espacio rural en América Latina, puede hablarse de una brecha de género, donde las relaciones hombre-mujer ocurren de manera desigual, en la que uno y otro, por lo general, se ocupan de tareas diferentes. Manifiestan Pérez y Fuller (2015), que sumar un enfoque de género a los proyectos, sin dudas influye en la autonomía, independencia económica, y posicionamiento de las féminas respecto a sus parejas, refiriéndose la autonomía desde su visión más abarcadora, aquella en la cual la mujer es partícipe también de los análisis de las agendas de desarrollo con base a sus derechos.

El turismo rural, según la World Tourism Organization (WTO, 2004) puede constituir una manera válida de promover la equidad de género, debido a las amplias posibilidades de empleo que proveen para grandes cantidades de mujeres en comparación con otras esferas, a la par que fomenta la creación de nuevos negocios de pequeña y mediana proporción. Así, la Organización Internacional del Trabajo (OIT, 2002), considera que podría hablarse de independencia financiera de las mujeres como medio para el mejoramiento de sus niveles de vida y autoestima, siendo resilientes en lo que desean.

El término resiliencia, emerge como fundamental también para poder entender el fenómeno a cabalidad. Al analizarse este tema exhaustivamente, sin dudas se afirma que se destaca por su carácter multidisciplinar. Oriol-Bosch (2012), explica que en el ámbito de las ciencias de la vida, se entiende como "la adaptación de la persona para vencer los 
obstáculos, las amenazas, permitiéndole vivir y desarrollarse positivamente o superarse, venciendo el estrés o las adversidades que se le presenten para afrontar desafíos, impulsando el desarrollo positivo" (p.77).

Por ello, una postura resiliente, sería esencial para las mujeres al enfrentarse con su verdadera autonomía, a pesar de lo complejo $\mathrm{y}$ adverso de un contexto social patriarcal y sexista, coincidiendo con Oriol-Bosch (2012), porque se puede establecer el vínculo entre autonomía, género y resiliencia, considerado, según los propios autores como la condición que expresa los disímiles mecanismos de adaptabilidad tanto de las personas como de los grupos sociales, se desdoblan también de formas diversas para enfrentar retos, amenazas $\mathrm{y}$ adversidades.

\section{Metodología}

Para el desarrollo del presente artículo como parte de la investigación, se asumió el enfoque interpretativo, con tipo fenomenológico, etnográfico, al situarse en el espacio geográfico, para a través de entrevistas, conocer y analizar la situación de la parroquia Crucita, del cantón Portoviejo, provincia ecuatoriana de Manabí, para fundamentar un proyecto de turismo en el espacio rural con enfoque de género que potencie el desarrollo local.

La obtención de información acerca del estudio, se logró con los informantes clave, hombres y mujeres de la localidad cuyo criterio de selección fueron: Ejecutan actividad gastronómica familiar; aquellos donde se observa que el hombre realiza la función pública en espacios que tienen presencia de féminas. Las técnicas cualitativas empleadas fueron la entrevista estructurada y en profundidad, así como la observación directa del fenómeno. Para interpretar los hechos se empleó como método, el análisis de contenido.

\section{Resultados y discusión}

Como parte del estudio, se identificó el espacio, indicándose que Crucita cuenta con 60 restaurantes que forman parte de un negocio familiar, de ellos 25 se encuentran ubicados en la zona del malecón, que recepcionan la mayor cantidad de visitantes y, por ende, obtienen mayores ingresos. Los demás restaurantes (35), se sitúan entre las calles transversales, la avenida principal y comunas. Estos son menos visitados por los huéspedes de los hostales ubicados en esa área, aunque poseen una venta más estable en horario nocturno.

A través de los métodos y técnicas descritos anteriormente, se pudo constatar que la gastronomía constituye la principal fuente de ingreso de la población de Crucita, pues el $50 \%$ de sus habitantes se dedica a esa labor como parte de la actividad turística. No obstante, constituye precisamente una de las manifestaciones más palmarias de la inequidad de género, pues la mujer elabora los alimentos, pero su papel se limita a la cocina, mientras el hombre se muestra como guía y dueño del negocio. Lo anterior se refuerza en el marco de la realidad de la mujer que habita en los espacios rurales, donde la influencia machista se encuentra más arraigada.

Varios testimonios obtenidos en las entrevistas dan cuenta de roles subordinados de las mujeres y de su presencia en labores etiquetadas como femeninas, considerándolas para actividades de limpieza, esa ha sido la costumbre en el lugar, y por ello, lo ven como un medio aceptable para obtener sus ingresos propios. Esto concuerda con la posición de Ferguson (2010), quien considera que el empleo en el sector turístico está marcadamente estructurado en función del género; las desigualdades globales favorecen una oferta de trabajadoras receptoras de muy bajos salarios. Tal como lo menciona una de las mujeres entrevistadas:

Hay un grupo de mujeres, tengo entendido, que ellas se han organizado como asociación de limpieza, se van a hacer limpieza en los hoteles, restaurantes, en casas, no solo dentro de la parroquia, sino que se van afuera también de la parroquia, 
en el área ya de la ciudad a trabajar también donde las llamen, entonces la mujer ya no se queda en casa, ya la mujer es parte de emprender, sí, de producir exactamente. (Entrevistada 1)

Ante esas dinámicas, resulta necesario potenciar el turismo y posicionar en la gastronomía como eje fundamental para el desarrollo local, lo cual debe hacerse desde actividades sociales más integradoras, que faciliten la participación de la mujer. En cuanto a la autonomía, vista desde el enfoque de género, en las entrevistas realizadas se evidencian síntomas de actitudes resilientes, en medio de un contexto adverso desde el punto de vista del desarrollo de la mujer, al querer demostrar sus habilidades culinarias como medio de trabajo. Coincide lo anterior con Oriol-Bosch (2012), en cuanto a que la resiliencia social depende de la confianza mutua y de la fortaleza de los vínculos en el grupo y expresa su capacidad de absorber las presiones y estabilizarse rápidamente. Así lo explica una de las entrevistadas:

La mujer está incursionando en diferentes áreas, aquí hay algunos grupos de mujeres que visceran el pescado, que trabajan para la pesca; hay otro grupo de mujer [es] que trabaja en el área turística, hay otro grupo de mujeres que trabajan en un grupo que se llama Santa Marta: ellas cuidan a niños, trabajan para las guarderías, trabajan en los $\mathrm{CNH}$, en los Sí bebé. Hay también otro grupo de mujeres que hacen catering de Crucita. La mujer hoy en día aquí en nuestra parroquia, en el área rural, está incursionando en muchas cosas, está emprendiendo. (Entrevistada 2)

En las informaciones recogidas, se pudo apreciar que, aun cuando se perciben cambios en la comunidad, todavía son sensibles de transformación, aspectos como los cánones que le asignan a la mujer la total responsabilidad del hogar, y por eso, muchas trabajan en el cuidado de los hijos de otros, porque es una actividad que saben hacer, considerándola un medio efectivo de producir ingresos. Así se deduce de la siguiente opinión:

Sí así es, la mujer se ha liberado hoy en día, sí porque ellas tienen la oportunidad de sus niños dejarlos en las guarderías. Entonces la mujer ya no se queda en casa se va a trabajar $\mathrm{y}$ pertenecen a algunas organizaciones $\mathrm{y}$ dentro de las organizaciones ellas están agrupadas para ejercer ciertos trabajos. (Entrevistada 3)

Una de las entrevistadas manifestó su deseo y el de otras mujeres de la localidad, "de montar un restaurante con la comida propia del lugar", a lo que se le preguntó cuáles eran los obstáculos, respondiendo: "Además, de necesitarse mucho dinero, tenemos que luchar con nuestros maridos porque ellos piensan que nosotras no estamos preparadas para eso". Destaca que existe una marcada desigualdad de género, a pesar de estar las mujeres, por lo menos una mayoría, dispuestas a trabajar y a tener su propio negocio, en función de sus habilidades culinarias, la mayor oposición la encuentran en sus maridos, situación que deben vencer.

Exponen Trejo, et al., (2015), en cuanto a que esta diferenciación se debe a la educación, la sociedad y la familia, las cuales han trasmitido valores, costumbres, estereotipos, prejuicios y roles sobre lo que supuestamente "debe desempeñar" cada género, de manera socialmente impuesta, aunque varían de una cultura a otra y de una a otra época que esté viviendo una sociedad, cuestión que en esta localidad está muy marcada.

Por otra parte, en varias entrevistas se constató que los hombres desconocían los elementos propios del concepto de autonomía o les resultaba indiferente, pues aseguraban que eran autónomos "per se", muestra del machismo aun intrínseco en la parroquia y en la región en general, al considerar al hombre como el único capaz de realizar los trabajos importantes. Uno de ellos expresó:

Los trabajos de Crucita son para nosotros los hombres, pescamos, servimos de guías a turistas extranjeros, les brindamos nuestra comida y mostramos lo que hacemos. Nuestras mujeres hacen la comida pero ellas están en la cocina y realmente (así lo dijo), nosotros quedamos bien ante los visitantes y ellas no se muestran. (Entrevistado hombre 1)

Otro hombre de Crucita al ser entrevistado, mostró una actitud de gerente, reconociendo las oportunidades que el sector 
le ofrece, coincidiendo esto con la posición de Monge y Macías (2016), en cuanto a la participación y la coordinación de gobiernos locales y otras instituciones sociales que comienzan a hacerse imprescindibles para lograr el cambio, y se produzca un compromiso real de la comunidad en el proceso de transformación local con sus playas y su comida. Al preguntarle que hacían ellos y que les correspondía a las mujeres, explicó:

Mis compañeros y yo tenemos como un equipo de trabajo, cuando llega la temporada alta que llegan turistas de aquí de Ecuador y de otras partes del mundo, nos distribuimos para servir de guías turísticos y mostrarles los lugares más bonitos de Crucita, y a las mujeres les toca cocinar, para eso están ellas. Eso sí, la comida que hacen es muy sabrosa y los visitantes quedan muy contentos y satisfechos. (Entrevistado Hombre 2)

Al preguntarle a uno de los hombres entrevistados si tenían un proyecto turístico para Crucita, respondió: "Queremos hacer algo bueno, estamos programándolo pero estamos en la búsqueda de asesoría porque vemos que este lugar es muy visitado, y queremos que lo que hagamos quede para acá, pero se necesita dinero, hay que invertir", lo cual indica el interés de ellos por desarrollar un turismo más programado, tal como debe ser y como explican Pérez, et al., (2020) que la percepción emocional, en relación con el patrimonio histórico visitado, se traduce en valoraciones significativamente distintas con respecto a los recursos turísticos del destino, de allí que deba tomarse en cuenta al tipo de turista que los visita, así como la necesidad de brindarle los servicios para que se sientan atraídos y agradados.

Estas entrevistas a la luz de los expertos en el área de desarrollo local, indican la posibilidad de producir en el espacio, concordando con Flores y Barroso (2012); así como con Loor, Plaza y Medina (2021), para quienes dentro de los factores de oferta se encuentra la actividad turística la cual comienza a ser considerada una alternativa que puede permitir una cierta recuperación socioeconómica ante la crisis de las zonas rurales.

De modo general, es posible arribar a importantes resultados a través del análisis de Debilidades, Amenazas, Fortalezas y Oportunidades (DAFO) (ver Figura I), como vehículo para realizar el diagnóstico de la situación de la parroquia Crucita, dirigido hacia las cuestiones relativas al enfoque de género, los retos y posibilidades que presenta la puesta en funcionamiento de un proyecto que tenga en cuenta la equidad entre ambos sexos.

Se obtuvo el diagnóstico de la situación pudiéndose detectar que según el DAFO realizado, existen grandes oportunidades para el desarrollo turístico local en Crucita con la posición de las mujeres, quienes a pesar de ser un sector disminuido en su valor por la cultura que prevalece, muestran una marcada tendencia a propiciar el crecimiento económico local, con posibilidades de empleo específicamente en la gastronomía, además, de estar interesadas en desarrollar sus planes de tener restaurantes y bebida que caracteriza la comunidad. 


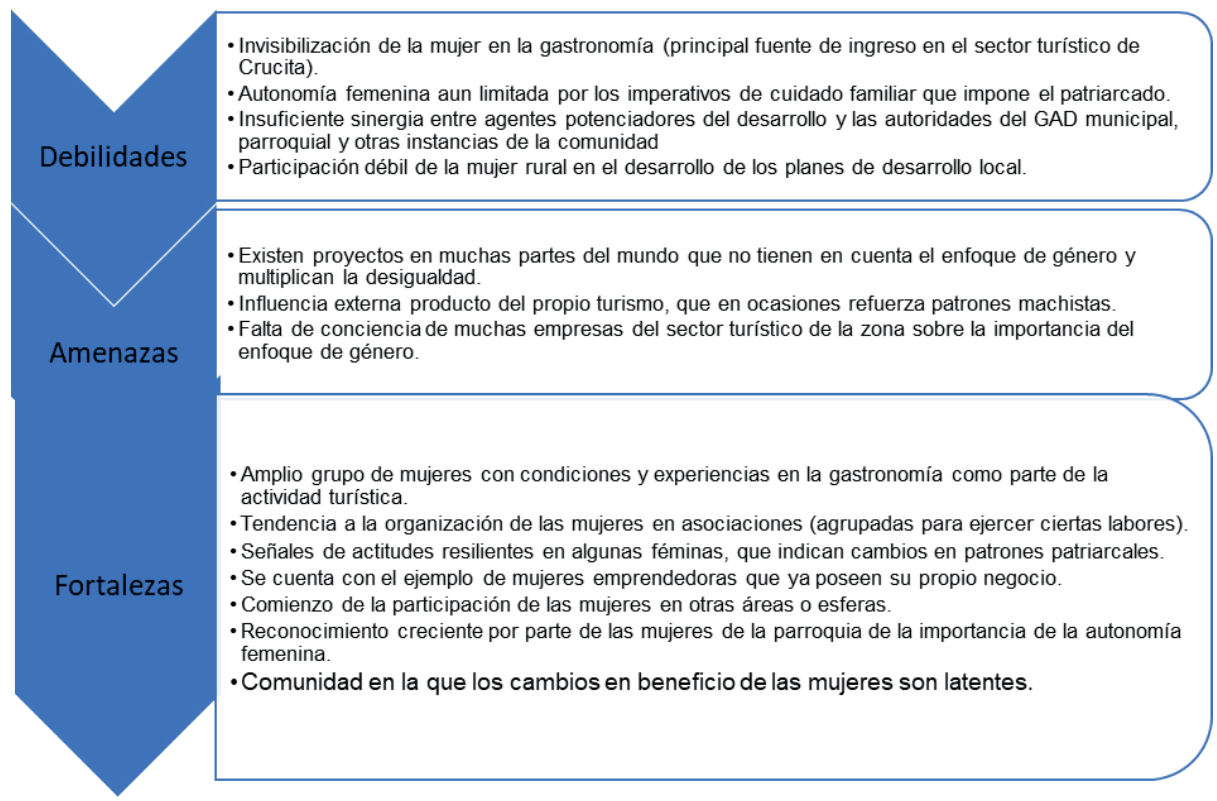

Fuente: Elaboración propia, 2020.

\section{Figura I: Análisis DAFO sobre la implementación de proyectos de desarrollo local en Crucita, cimentados en el turismo y con enfoque de género}

Este interés resiliente de muchas mujeres de Crucita, evidencia posibilidades de realizar sus propios negocios que a la par de contribuir con el crecimiento del lugar, permite la estructuración de esa playa con nuevos empleos y oportunidades. No obstante, deben vencer la oposición que encuentran en sus maridos y hombres de la localidad, como principio de acción para lograr lo que necesitan y quieren.

\section{Conclusiones}

En el presente artículo se trataron cuestiones referentes al desarrollo local y el turismo rural desde un enfoque de género, por darle gran relevancia al papel de la mujer como motor dinámico del turismo en Crucita, Ecuador, y a partir de lo anterior, se pudo obtener una visión más integral del fenómeno y aprehender también nociones relativas a la resiliencia y la autonomía femenina.

Al analizar el diagnóstico de la parroquia Crucita, se evidencia que es una comunidad que muestra avances en lo referente a las cuestiones de género, desde el punto de vista de la autoestima y autonomía de la mujer, lo cual se manifiesta, en una red de cooperación y participación en torno a proyectos colectivos y en la deconstrucción de cánones patriarcales trasmitidos de una generación a otra, por solo mencionar algunos ejemplos. Por ello, resultan necesarios cambios para lograr la verdadera equidad, y una muestra fehaciente de ello es la actividad gastronómica.

Se expresa además, que el crecimiento del turismo en el espacio rural, deviene elemento esencial para fomentar el desarrollo local y si se diseñan correctamente proyectos 
a tal efecto y que tengan en cuenta una perspectiva de género, será posible entonces contribuir desde disímiles maneras a la autonomía femenina, que junto a la resiliencia se articulan en el entorno rural de Crucita en Ecuador.

De modo general, se reconoce la importancia de implementar en la localidad iniciativas donde la mujer promocione sus talentos dentro del área de la gastronomía, propiciando así su participación social al considerar las características propias del ambiente rural, donde las actitudes conservadoras y los rezagos sexistas resultan aún más fuertes que en la ciudad.

\section{Referencias bibliográficas}

Alonso, J.; Riera, C. y Rivero, R. (2013). Fundamentos conceptuales y metodológicos del autodesarrollo comunitario como alternativa emancipadora. Editorial Feijoo.

Asamblea Nacional (2011). Constitución de la República del Ecuador 2008. Registro Oficial 449 de 20-oct-2008. Ultima modificación: 13-jul-2011. https:// www.oas.org/juridico/pdfs/mesicic4 $\underline{\text { ecu const.pdf }}$

De Oliveira, E., De Souza, A., Scherer, L., Thome, H., y Spindler, M. M. (2014). El papel del turismo en el espacio rural de São José dos Ausentes, Brasil. El Periplo Sustentable, (27).

Ferguson, L. (2010). Turismo, igualdad de género $\mathrm{y}$ empoderamiento de las mujeres en Centroamérica. Papeles de Relaciones Ecosociales y Cambio Global, (111), 123-133.

Flores, D., y Barroso, M. D. L. O. (2012). El turismo como estrategia de desarrollo rural sostenible. Los parques naturales andaluces. Revista de Estudios Empresariales. Segunda Época, (1),
59-83.

Formichella, M. (2004). El concepto del Emprendimiento y su relación con la educación, el empleo y el desarrollo local. Instituto Nacional de Tecnología Agropecuaria.

García, C. (2016). Turismo comunitario en Ecuador: ¿Quo vadis? Estudios y Perspectivas en Turismo, 25(4), $597-$ 614.

Instituto Internacional de Investigaciones y Capacitación de las Naciones Unidas para la promoción de la Mujer - INSTRAW (2006). Glosario. INSTRAW. http://www.un-instraw. org/es/

Instituto Nacional de Estadística y Censo INEC (2010). Resultados del Censo 2010 de Población y Vivienda en el Ecuador. Fascículo Provincial Manabí. INEC. https://www.ecuadorencifras. gob.ec/wp-content/descargas/Manulateral/Resultados-provinciales/ manabi.pdf

Loor, L., Plaza, N., y Medina, Z. (2021). Turismo comunitario en Ecuador: Apuntes en tiempos de pandemia. Revista de Ciencias Sociales (Ve), $X X V I I(1), \quad 265-277 . \quad \underline{h t t p s}$ ://doi. org/10.31876/rcs.v27i1.35312

Millán, G., Morales, E., y Pérez. L. (2014). Turismo gastronómico, denominaciones de origen y desarrollo rural en Andalucía: Situación actual. Boletín de la Asociación de Geógrafos Españoles, (65). https:// doi.org/10.21138/bage. 1746

Monge, Y. V., y Macías, A. R. (2016). El desarrollo local. Enfoques para su conceptualización. Revista Científica Olimpia, 13(39), 226-238.

Organización Internacional del Trabajo - OIT (2002). Gestión del turismo sostenible y competitivo. Alianzas entre Estado, empresa y comunidad. Los casos 
de Bolivia, Ecuador y Perú. OIT. https://asesoresenturismoperu.files. wordpress.com/2016/04/129-omtgestion-de-turismo-sostenible.pdf

Organización Mundial del Turismo - OMT (2019). Barómetro OMT del Turismo Mundial y anexo estadístico, Enero 2019. OMT. https://www.eunwto.org/doi/abs/10.18111/ wtobarometeresp.2019.17.1.1

Oriol-Bosch, A. (2012). Resiliencia. Educación Médica, 15(2), 77-78.

Pacheco, V., Carrera, P., y Almeida, K. (2011). Propuesta metodológica para la evaluación de la factibilidad de proyectos de turismo comunitario. Caso de estudio: Comunidades Huaorani, Achuar y Shiwiar de la Amazonia Ecuatoriana. Gestión Turística, (15), 21-46. https://doi. org/10.4206/gest.tur.2011.n15-02

Pelegrín, N., Martínez, O., Pelegrín, A., y Zaballa, E. L. (2020). Senderismo como opción para pequeñas ciudades patrimoniales en regiones turísticas: Trinidad de Cuba. Revista de Ciencias Sociales (Ve), XXVI(3), 231243. https://doi.org/10.31876/rcs. v26i3.33244

Pérez, B., y Fuller, N. (2015). Turismo rural comunitario, género y desarrollo en comunidades campesinas e indígenas del sur del Perú. Quaderns de l'Institut Catalá d'Antropologia, (31), 95-119.

Pérez, J. C., Medina-Viruel, M. J., LópezGuzmán, T., y Muñoz-Fernández, G. (2020). Segmentación y percepción turística en destinos patrimonio material de la humanidad: Córdoba (España). Revista de Ciencias Sociales (Ve), XXVI(1), 11-24. https://doi. org/10.31876/rcs.v26i1.31307

Programa de las Naciones Unidas para el Desarrollo-PNUD(2006). Reflexiones. Desarrollo local con equidad de género. PNUD. https://dhls.hegoa. ehu.eus/uploads/resources/4787/ resource files/Reflexiones sobre desarrollo local con equidad de g\% $3 \%$ A 9 nero 2006 . pdf? $v=63735876601$

Rodríguez, M. (2011). Plan de acciones para fortalecer la promoción de la mujer a cargos de dirección en la Fábrica de Tabaco Juan Casanueva del Municipio de Consolación del Sur (Tesis de pregrado). Universidad de Pinar del Río, Cuba.

Secretaría Nacional de Planificación y Desarrollo - SENPLADES (2015). Plan de Desarrollo y Ordenamiento Territorial: Parroquia Crucita 2015. SENPLADES https://bit.ly/2R4F4MU

Segrelles, J. A. (2015). Conceptos básicos sobre desarrollo local y sostenibilidad. Geografía y desarrollo local. Universidad de Alicante, España. https://rua.ua.es/dspace/ bitstream/10045/48107/1/Tema 1 $\underline{\text { DLS.pdf }}$

Serrano, P. (2012). La perspectiva de género como una apertura conceptual y metodológica en salud pública. Revista Cubana de Salud Pública, 38(S-5), 811-822.

Tezanos, S., Quiñones, A., Gutierrez, D., y Madrueño, R. (Coord.) (2013). Manuales sobre cooperación $y$ desarrollo. Desarrollo humano, pobreza y desigualdades. CLACSO. Consejo Latinoamericano de Ciencias Sociales.

Trejo, M. L., Llaven, G., y Pérez, H. C. (2015). El enfoque de género en educación. Atenas, 4(32), 49-61.

Verdesoto, L., y Ardaya, G. (2011). Continuidad y cambios en el desarrollo local y regional. Una aproximación al caso ecuatoriano. Polémika, 2(6), 130143. 
Turismo rural en Crucita-Ecuador: Una mirada desde la fortaleza del género

World Tourism Organization - WTO (2004). Zambrano, M., Alejo, O. J., y Zambrano, P. Rural tourism in Europe: Experiences, development and perspective. WTO. https://www.e-unwto.org/doi/ $\underline{\mathrm{abs} / 10.18111 / 9789284407163}$ (2019). Factores influyentes en la lealtad electrónica hacia comunidades turísticas en línea: Caso mochileros. Revista de Ciencias Sociales (Ve), $X X X V(3), 200-217$. 